\title{
Effect of Functional Motivations of Volunteers on Participation Satisfaction and Sustainability
}

\author{
Nam-kyo Yun1), Sung-je Cho2)
}

\begin{abstract}
The purpose of this study is to identify the effects of the functional motivations of volunteers on participation satisfaction and sustainability. The study was conducted on 198 volunteers in Seocho-gu, Seoul. For the analysis method, frequency analysis, reliability analysis, and correlation were performed using the SPSS 22.0 program, and regression analysis was performed on causality among variables. The study found that the functional motivations for volunteers to do volunteer work have a significant effect on their satisfaction with volunteer work. Second, the functional motivations for volunteers to do volunteer work have been found to have a positive effect on the sustainability of volunteer work. This can be said to be a positive tool in increasing the level of participation satisfaction, will to continue, and intensity for participation in volunteer work, such as self-protection, value-seeking, and social skills, which are the functional motivations for volunteers to do volunteer work. Therefore, it is thought that this study will be the basis for preparing programs to satisfy volunteers' participation in volunteer activities and to enhance their sustainability.
\end{abstract}

Keywords: Volunteer, Functional Motivation Factor, Participation Satisfaction, Sustainability, Intensity for Participation

\section{Introduction}

\subsection{Necessity of Research}

Recently, social welfare policies have been implemented to intervene and solve social problems caused by the aging of the population and low birth rate at the national level. On the other hand, despite the state's implementation of social welfare policies, there are still second-class people who suffer in blind spots due to a lack of budget and public manpower. Therefore, as interest in volunteers emerges, research on the effects of functional motivation factors on

Received(April 7, 2020), Review Result(1st: May 11, 2020, 2nd: June 25, 2020), Accepted(July 27, 2020)

1) (Ph.D. course/doctorate course) 02838 Dept. of Culture and Arts Contents, Dongbang Culture Graduate Univ., 28 Sungbok-Ro, Sungbokdong, Seoul, Korea

email: fy2001@naver.com

2) (Professor, Corresponding Author) 02838 Dept. of Culture and Arts Contents, Dongbang Culture Graduate Univ., 28 Sungbok-Ro, Sungbokdong, Seoul, Korea

email: chosj715@daum.net 
participation satisfaction and persistence are required. First It can be said that activating volunteer work is necessary to address these social problems[1]. As such, volunteer work is a way to support those in second-class, which can not be solved only by the nation's social welfare policies.

Volunteer activities are activities that "continue to devote time and effort voluntarily without expecting specific compensation for the promotion of the welfare of the public"[2], and these volunteer workers are called volunteers. Volunteers can volunteer at any time, at any place, and in any way by their own free will[3]. Given these characteristics of a volunteer's free will, unless there is an environment in which the participating volunteers can continue to serve, an increasing number of volunteers will give up their volunteer service in the middle[4]. Therefore, research is needed on various factors that determine sustainability to prevent volunteers from giving up their jobs to continue to do volunteer work[5].

Sustainability of volunteering is a social product of the rewards of volunteering as a motivation for participation by volunteers[6], and the sustainability of volunteers will decrease when the service is not as rewarding as one expects. Generally, factors that determine sustainability can be divided into individual and institutional characteristics, and in order to review the factors related to the sustainability of volunteer activities, we will first review the relationship between functional motivations and participation satisfaction and sustainability in this study. Volunteers are not people who do volunteer work in anticipation of remuneration. They should be those who have internal motivation (functional motivation) to voluntarily participate in the service activities to gain satisfaction from the volunteer work, to continue the volunteer work, and to participate in the service organization over the long term.

Therefore, it is expected that a person with a high functional incentive to attract to volunteer activities will be more satisfied with the volunteer work and that performance (will to continue the activity, pro-social behavior) will be higher[7]. Meanwhile, it was confirmed that the stronger the motivation for internal participation, the higher the satisfaction and self-esteem of the volunteers[8]. Therefore, it is considered that the functional motivations of volunteers can affect their participation satisfaction and sustainability, so I want to look into them.

Studies on the factors affecting the satisfaction and sustainability of volunteer work include the following. First of all, Ya-gi Yang[9] found that the motivation for participating in volunteer work has been found to affect sustainability. Therefore, it suggests that it is necessary to improve the motivation for participating in volunteer work to improve the sustainability of volunteer work. 
Mi-ryeong Kim[10] saw the nature of volunteer work and motivation for participation as factors that affect volunteer satisfaction. In summing up the above discussions, most of the factors affecting the satisfaction of volunteer work are the motivation, interpersonal relationships of volunteers and characteristics of volunteer work. However, there has been no study on factors affecting participation satisfaction in terms of the functional motivations of volunteers.

Therefore, I want to identify functional motivations and relationships of participation satisfaction and sustainability among volunteers, and to identify factors that affect the sustainability of volunteer activities. Therefore, the purpose of this study is as follows.

First, it aims to grasp the relationship between functional motives. Second, the factors affecting the satisfaction and sustainability of volunteer participation and the direction of volunteer activity are analyzed. Third, it provides the basic materials necessary to strengthen the direction of improvement of volunteer activities and develop alternatives to continuously participate in volunteer activities

\subsection{Research Problem}

The purpose of this study is to analyze the effects of the functional motivations of volunteers on their satisfaction and sustainability. Specific research issues for this are as follows.

First, what is the effect of functional motivations for volunteers on their satisfaction with volunteer work?

Second, what is the effect of the functional motivations of volunteers on the sustainability of volunteer work?

\section{Research Method}

\subsection{Research Subjects}

The purpose of this study was to examine the effect of volunteers' functional motivation on the persistence of volunteer activity in relation to the effect of volunteers' functional motivation on participation satisfaction and persistence. Therefore, from March 1, 2019 to June 30, 2019, a questionnaire was distributed to 215 volunteers who live in Seocho-gu, Seoul, and participate in volunteer work. After sufficiently explaining the purpose, it was carried out by a self-writing method. A total of 208 copies of the study data were collected, showing a return rate of $96.7 \%$, and a total of 198 copies of the collected questionnaires, except for 10 unsuitable 
questionnaires, were analyzed and used in the study.

\subsection{Research Tool}

The tools used in this study consist of 5 general characteristics, 30 functional motivations, 7 participatory satisfaction levels, and 6 sustainability questions. Contents concerning general characteristics were investigated by organizing gender, age, academic background, religious status, and types of volunteer activity organizations. To measure the functional motivations of volunteers, the measuring tools for functional motivations used by Jun-mok Kim[11] comprised a total of 30 questions and measured each question on a five-point scale of Likert. To measure volunteers' participation satisfaction, Chun-hee Park's participation satisfaction measurement tool[6] was used to measure the level of satisfaction with participating in volunteer activities on a five-point scale of Likert. To measure the sustainability of volunteers, Chun-hee Park[12]'s tools for measuring sustainability were used to measure the level of sustainability of volunteers and the intensity of participation in the future, measured on a five-point scale of Likert.

\subsection{Reliability}

The reliability verification of each variable used in this study shows that the functional motivation is .914 and the reliability of the participating satisfaction is .814, and the reliability of sustainability is .792. The reliability of each measuring tool is ensured to be higher than the minimum of Cronbach's a value, .60. The reliability analysis results of the major variables are shown in [Table 1].

[Table 1] The Reliability of the Major Variables

\begin{tabular}{ccc}
\hline Sortation & Questions & Cronbach's $\alpha$ \\
\hline Functional motivations & 22 & .914 \\
Participation satisfaction & 7 & .814 \\
Sustainability & 6 & .792 \\
\hline
\end{tabular}

\subsection{Data Processing and Analysis Method}

The data collected in the study was verified at the significance level of $5 \%$ using the SPSS 22.0 
program, and the reliability calculation of each research tool was analyzed using a coefficient of Cronbach's a. The demographic characteristics of volunteers used frequency analysis, correlation analysis was conducted on functional motivations, participation and sustainability of volunteers and a regression analysis was performed to identify causality.

\section{Results}

\subsection{Demographic Characteristics}

Looking at the demographic characteristics of the subjects in this study, the gender of the job-seekers was found to have a higher percentage of women's responses, with 88 men $(44.4 \%)$ and 110 women (55.6\%). Those in their 50s accounted for the largest share of 69 people (34.8 percent), followed by 44 people in their 40s (22.2 percent), 36 people in their 60s (18.2 percent), 30 people in their 20s or younger (15.2 percent), and 19 people in their 30s (9.6 percent). The highest number of college and college graduates was 99 (50.0 percent), followed by 52 high school graduates (26.3 percent) and 47 graduates from graduate school (23.7 percent). 123 people with religion (62.1 percent), and 75 people without religion (37.9 percent), were found to be more subjects with religion. The survey found that the number of social welfare facilities for senior citizens was 118 (59.6 percent), and 80 (25.1 percent) for counseling services (children, teenagers). The study found that the type of social welfare facilities where subjects do volunteer work has more facilities for the elderly.

\subsection{Technical Statistics on Major Variables}

[Table 2] Technical Statistics on Major Variables

\begin{tabular}{cccccc}
\hline Sortation & $\mathrm{N}$ & $\begin{array}{c}\text { Minimum } \\
\text { value }\end{array}$ & $\begin{array}{c}\text { Maximum } \\
\text { value }\end{array}$ & $\mathrm{M}$ & $\mathrm{SD}$ \\
\hline Functional motivations & 198 & 1.91 & 5.00 & 3.28 & .68 \\
Participation satisfaction & 198 & 1.14 & 5.00 & 3.56 & .76 \\
Sustainability & 198 & 1.00 & 5.00 & 3.43 & .72 \\
\hline
\end{tabular}




\subsection{Correlation between Variables}

[Table 3] Correlation between Major Variables

\begin{tabular}{cccc}
\hline Sortation & Functional motivations & Participation satisfaction & Sustainability \\
\hline Functional motivations & 1 & & \\
Participation satisfaction & $.659^{* *}$ & 1 & 1 \\
Sustainability & $.687^{* *}$ & $.843^{* *}$ & \\
\hline${ }^{*} \mathrm{p}<.05,{ }^{* *} \mathrm{p}<.01$ & & &
\end{tabular}

3.4 Effect of Functional Motivations of Volunteers on Participation Satisfaction and Sustainability

\subsubsection{Effect of Functional Motivations of Volunteers on Participation Satisfaction and Sustainability}

A regression analysis was conducted to find out the effect of the functional motivations of volunteers on their participation satisfaction. The analysis results show that the R2 value, which refers to the descriptive power of functional motivations for the participation satisfaction of volunteers, is .435 , explaining the regression model at $43.5 \%$ of the total variation. The ANOVA also found that the regression formula was suitable and statistically significant at $\mathrm{F}=150.822(\mathrm{p}<.000)$. When looking at the influence of functional motivations, the functional motivations $(\beta=.659, \quad \mathrm{p}<.001)$ was analyzed to have a positive $(+)$ effect on participation satisfaction at a statistical significance level. This means that the higher the functional motivations of volunteers, the higher the level of participation satisfaction.

\subsubsection{Effects of Functional Motivations on Sustainability of Volunteers}

A regression analysis was performed to find out the effect of the functional motivations of volunteers on their sustainability. The analysis shows that the R2 value, which refers to the explanation of functional motivations on the sustainability of volunteers, is .472 , describing the regression model at $47.2 \%$ of the total variation. In addition, in ANOVA, $F=175.350(p<.000)$ indicates that the regression formula is suitable and statistically significant. When looking at the influence of functional motivations, the functional motivations $(\beta=687, p<.001)$ was analyzed to 
have a positive effect on sustainability at a statistical significance level. This means that the higher the functional motivations of volunteers, the higher the sustainability.

\section{Conclusion and Suggestion}

In this study, the effects of functional motivations of volunteers on participation satisfaction and sustainability were analyzed. Based on the analysis results, the detailed discussion is as follows.

First, the functional motivations for volunteers were found to have a positive effect on their participation satisfaction with volunteer work. This means that the higher the functional motivations of volunteers, the higher the level of participation satisfaction while doing volunteer work.

Second, the functional motivations for volunteers to do volunteer work were found to have a positive effect on the sustainability of volunteer work. This means that the higher the functional motivations of volunteers, the higher the sustainability of volunteer work.

Third, self-protection, value pursuit, sociality, etc., which are the functional motivations for volunteers to volunteer, meaning that they are positive factors in increasing participation satisfaction and participation intensity after volunteer activities.

As discussed above, there is a growing need for volunteer work to solve various social problems and promote a sense of community at the same time in terms of social welfare services for volunteers, so the level of participation satisfaction and sustainability of volunteer work is very important. It is necessary to develop various educational programs and study contents for volunteers. Although there have been studies on the effects of the motivation, satisfaction of volunteer work, and will to sustain volunteer work in relation to volunteer work of volunteers, this study is meaningful in that it has identified the effects of functional motivations on sustainability and analyzed that functional motivations have an impact on participation satisfaction. Based on these findings, it is necessary for volunteers to develop programs such as systematic support for the participation satisfaction and programs that can increase the sustainability of volunteer work. In the future, in-depth research is required on the effectiveness of functional motivations for volunteers. 


\section{References}

[1] Yoon-ro Lee, Mi-sook Cho, Utilization of Volunteers in Social Welfare Institutions and Tasks, Korean Regional Social Welfare Studies, (2004), Vol.14, pp.189-215.

[2] Myeong-rye Kang, A Study on the sustainability Factors of Youth Volunteer Activity: a doctoral dissertation focusing on high school students in Yeoju, Hyup Sung University, Doctoral Dissertation, (2019)

[3] Jung-hun Kim, Analysis of Influential Factors on the Sustainability of Volunteer Activities- Focused on Daegu Citizens, Local Government Research, (2010), Vol.14, No.3, pp.117-138.

[4] Geum-dan Sung, Nam-yeon Cho, Analysis of the Influence Factors on the sustainability and Satisfaction of Volunteer Activities, Humanities Society 21, (2018), Vol.9, No.2, pp.791-800.

[5] Yong-sik Yoo, Ho-jung Son, The primary factors affecting durability of volunteering, KOREAN NPO REVIEW, (2009), Vol.8, No.2, pp.27-57, UCI : G704-001881.2009.8.2.004

[6] Chun-hee Park, A Study on the Factors Influencing the Volunteer Activities of Citizens, Konkuk University, Doctoral Dissertation, (2015)

[7] In-duk Seo, Jun-mok Kim, A Study on the Impacts of Volunteers's Functional Motives and Managerial Practices of Volunteers Administration Centers on Center's Organizational Performance-Focused on the Mediating Role of Volunteers Satisfaction, Journal of Business Research, (2012), Vol.27, No.1, pp.99-129, DOI : 10.22903/jbr.2012.27.1.99

[8] Bong-kyu Park, Jae-wan Cho, The effect of volunteer motivation on satisfaction and self-esteem, Tourism Research, (2004), Vol.18, No.3, pp.143-157.

[9] Ya-gi Yang, The Influence of Nursing University Student's Motivation for Volunteer Activities and Satisfaction on the Intention of Volunteer, Journal of Nursing Education, (2018), Vol.24, No.4, pp.415-423.

[10] Meeryoung Kim, The effect of baby boomer volunteers' volunteering and the motives of volunteering on the volunteer role identity and volunteer satisfaction, Korean Journal of Gerontological Social Welfare, (2014), No.64, pp.79-103.

[11] Jun-mok Kim, A Study on the Determinants of Retention and Pro-social Orientation of Volunteers :Focused on the Mediating Role of Voluntary Satisfaction, Yeungnam University, Doctoral Dissertation, (2012)

[12] Chun-hee Park, A Study of the Factors Affecting Citizens' Sustainable Partnership in Voluntary Activities, Konkuk University, Doctoral Dissertation, (2015) 